\title{
Características socioafectivas de niños con altas capacidades participantes en un
}

\section{programa extraescolar}

\section{Socioaffective characteristics of gifted children participating in an extracurricular program}

\author{
Gabriela López-Aymes*, Nancy Vázquez*, Martha Itzel Navarro*, Santiago Roger Acuña** \\ *Universidad Autónoma del Estado de Morelos, México **Universidad Autónoma de San Luis Potosí, México
}

\begin{abstract}
Resumen
Entre los estudios sobre las características socioafectivas del alumnado con altas capacidades, se pueden encontrar dos enfoques. En el primero se afirma que estos alumnos afrontan mejor los conflictos personales y sociales que sus iguales sin altas capacidades. En el segundo se aporta evidencia de que estos alumnos están en riesgo de padecer problemas emocionales y sociales. En ese sentido, el objetivo de esta investigación es conocer las características socio-afectivas que presentan niños con altas capacidades al inicio de un programa de enriquecimiento extracurricular de corte socioafectivo, con el fin de orientar la atención educativa.

Palabras clave: altas capacidades, habilidades sociales, intervención educativa.
\end{abstract}

\begin{abstract}
Among the studies on the socio-affective characteristics of gifted students, you can find two approaches. The first states that these students cope better personal conflicts and social tan peers without higher capacities. The second evidence that these students are at risk for emotional and social problems is provided. In that sense, the objective of this research is to understand the socio-affective characteristics that gifted children have at the beginning of a school enrichment program of social and emotional court, in order to direct educational services.

Keywords: gifted, social skills, educational intervention.
\end{abstract}

La investigación acerca de los niños y niñas con las altas capacidades ha cobrado mayor interés dentro del campo educativo. Dentro de los estudios consultados se ha encontrado que un número amplio de ellos se dedica a la conceptualización, la identificación e intervención educativa; así mismo, se destacan aquellos que se centran en la necesidad de conocer y potenciar el desarrollo integral de un niño o una niña con alta capacidad en todos los ámbitos donde se encuentre inmerso (Comes, Díaz, Luque \& Moliner, 2008). A pesar de poseer características singulares que los podría hacer destacar en cualquier área del desarrollo humano, encontramos que esta población puede enfrentarse a una serie de problemas. Narea, Lissi \& Arancibia (2006), manifiestan que los niños con altas capacidades están en riesgo de limitar sus habilidades durante el desarrollo, debido a las creencias y mitos acerca de ellos. Castro (2008) y López (2008) hacen referencia a los principales mitos sobre niños con altas capacidades, tales como la consideración que nunca tienen dificultades en la escuela, que siempre obtienen buenas calificaciones, que son personas retraídas, que no necesitan la ayuda de nadie, que son genios, que siempre son superiores físicamente, por mencionar solo algunos. Estos mitos han ido disminuyendo gracias a las investigaciones que se realizan acerca de esta temática, pero aún están presentes en gran parte de la población.

En ese sentido, es importante considerar el trabajo de forma multidimensional para la atención educativa de los alumnos con altas capacidades, con el objetivo de satisfacer todas las necesidades personales y sociales.

Particularmente, el estudio sobre el desarrollo de las áreas socio-afectivas de los niños y niñas con altas capacidades está ocupando un interés importante dentro de la investigación. Sin embargo, no está exento de controversias. Sobre todo en el área social, específicamente en la interacción con el otro.

La interacción se ha definido como aquella capacidad que posee el ser humano para establecer y consolidar relaciones con los otros (Flores \& Santoyo, 2009). A su vez, forma parte importante en la adquisición de habilidades, destrezas, actitudes y roles en el desarrollo y para la vida adulta (Clemente, Regla, Górriz \& Villanueva, 2003; Díaz- Aguado, 1986; García, 1993; Goldrine \& Rojas, 2007). En esta investigación se ha considerado que la interacción entre pares es clave en el desarrollo de estrategias sociales, sobre todo en la negociación, solución de problemas, adquisición de roles y relación con aspectos afectivos en los niños (DíazAguado, 1986).

En el ámbito educativo es posible observar las dificultades en la interacción en las actividades dentro del aula, sobretodo en el trabajo en equipo, el trabajo cooperativo, la formación de círculos sociales y la adopción de roles (López, 2008). Con respecto a los niños con altas capacidades, se ha hecho evidente que ellos pueden mostrar dificultades en la interacción, manifestándose, por ejemplo, por medio del aislamiento, el rechazo de sus compañeros, la dificultad de trabajar en equipo, una escaza participación dentro del aula, dificultades con la convivencia y dificultades en la formación de relaciones de amistad (Guzmán, 2010).

En algunos estudios como el de Vialle, Heaven \& Ciarrochi (2007), se comparó la perspectiva que tienen los alumnos con altas capacidades sobre sí mismos, y la perspectiva que tienen los docentes acerca de estos alumnos. El estudio arrojó que los alumnos con altas 
capacidades se consideraban a sí mismos con estándares afectivos más bajos en comparación del resto de la población y con mayor vulnerabilidad al enfrentar problemas. En contraparte, los docentes consideraban que los alumnos con altas capacidades eran menos propensos a tener problemas emocionales, ya que su aspecto académico compensaba los problemas que llegasen a tener. Ante esta discrepancia, se llegó a la conclusión de que los docentes deben prestar mayor atención a los alumnos con altas capacidades, principalmente en las áreas sociales y afectivas.

Por otro lado, Gómez \& Valadez (2010) afirman que los niños con altas capacidades prefieren jugar con compañeros mayores a ellos por el hecho de sentirse mejor que con sus compañeros de la misma edad. De igual forma, estas autoras han encontrado que los niños con altas capacidades suelen sentirse presionados por las expectativas de sus padres acerca de su comportamiento y futuro.

También Lovecky (1995) en sus investigaciones ha encontrado que los niños con altas capacidades manifiestan una escasa participación en el trabajo en equipo, ideas de superioridad y una perspectiva negativa hacia sus compañeros, por sentirse menos populares e incomprendidos por los niños de su edad. Lo anterior se ha explicado como una posibilidad de discrepancia en las áreas sociales y cognitivas. En esta misma vertiente, Roedell (2011) ha encontrado que los niños con altas capacidades sienten frustración cuando su esfuerzo no es reconocido o subestimado, por lo que puede derivar en una falta de confianza y autoconocimiento y miedo a relacionarse con los otros por las burlas o expresiones negativas hacia ellos. Por tanto, sugiere una investigación y trabajo en estas necesidades detectadas con el fin de atenderlas de forma inmediata.

Por otro lado, Díaz- Aguado (1986) señala que los niños que tienen dificultades en la interacción con sus pares tienen una probabilidad mayor a ser privados del desarrollo de competencias y habilidades sociales y afectivas, por tanto, la intervención dentro de estos aspectos debe ser un punto relevante para investigadores y sectores educativos. Así mismo, Ferrándiz, Prieto, Fernández, Soto, Ferrando \& Badía (2010) consideran la importancia de la atención en las interacciones sociales de estos alumnos, con el propósito prevenir posibles desajustes mediante la creación de programas. Estos programas deberán fomentar las habilidades sociales y afectivas en los niños con altas capacidades, considerando de forma permanente el trabajo con áreas cognitivas.

Es así, que se pueden encontrar dos posturas en cuanto al estudio de las interacciones de los niños con altas capacidades y la relación con sus pares. La primera postura, establece que los niños con altas capacidades llevan un desarrollo social igual o similar al de sus pares. Por ejemplo, en investigaciones llevadas a cabo por Borges, Hernández-Jorge \& Rodríguez-Naveiras (2011) y Hernández \& Borges (2010), encontraron que no existen diferencias en el ámbito de habilidades sociales entre niños de diferentes capacidades. Es decir, la interacción social entre pares es similar al resto de los niños, dándose de forma cooperativa, satisfactoria y exitosa. Asimismo, la probabilidad de presentar problemas sociales llega a ser mínima. La segunda postura, afirma que los niños con altas capacidades se encuentran desfasados en comparación con los niños de su edad, es decir, que llegan a presentar problemas. Belda (2012), Benito (2000), Flanagan \& Arancibia (2005) y López (2008) afirman que los niños con altas capacidades presentan algunas dificultades en el ámbito social; por ejemplo, dificultades en la comunicación, sentimientos de inferioridad y dificultad en la relación con su grupo. En consecuencia, Flanagan \& Arancibia (2005) manifiestan que los niños con altas capacidades llegan a presentar una asincronía evolutiva en el desarrollo, principalmente en las áreas cognitivas, sociales, y afectivas.

Los programas extracurriculares cumplen una función de enriquecimiento y complemento a la educación formal, es decir, no los sustituye, sino que contribuye al desarrollo y optimización de múltiples habilidades de los niños y niñas; al mismo tiempo, son una alternativa para cubrir sus necesidades, incorporando, desde una perspectiva social, a la familia y a los pares (Sartre-Riba, 2014)

En ese sentido, el objetivo de esta investigación es conocer las características socio-afectivas que presentan niños con altas capacidades al inicio de un programa de enriquecimiento extracurricular de corte socioafectivo (Programa Integral Para Altas Capacidades-PIPAC, México), con el fin de adecuar las estrategias educativas a las necesidades que presenten. El PIPAC tiene como objetivo principal contribuir al desarrollo integral de niños y niñas con altas capacidades, a nivel cognitivo, socioafectivo y comportamental, y se implementa en la Universidad Autónoma del Estado de Morelos (México) por especialistas en el tema.

\section{Método}

\section{Participantes}

En este estudio participaron cinco niños detectados con altas capacidades intelectuales en el Municipio de Cuernavaca, Morelos (México), en edades comprendidas entre los 8 y 12 años de edad.

Los criterios de inclusión que se consideraron dentro de esta investigación son:

1. Haber sido detectados previamente con altas capacidades intelectuales (percentil 85 o superior)

2. Estar escolarizados en escuelas públicas o privadas de Cuernavaca, Morelos.

3. Compromiso de participar durante un año escolar, tanto niños, niñas y padres de familia.

4. No participar en otro programa extracurricular similar mientras participa en el PIPAC.

\section{Instrumentos}

Para valorar las habilidades socioafectivas, se utilizó el BASC (Sistema de evaluación de la conducta de niños y adolescentes de Reynolds y Kamphaus, 2004). El BASC es un sistema de evaluación multidimensional que permite evaluar los aspectos adaptativos e inadaptativos de la conducta de niños y adolescentes. Evalúa aspectos 
positivos (liderazgo, habilidades sociales, habilidades para el estudio, adaptabilidad, relaciones interpersonales, relaciones con los padres, autoestima y confianza en sí mismo) como los negativos (ansiedad, agresividad, problemas de atención, problemas de aprendizaje, hiperactividad, retraimiento, búsqueda de sensaciones, problemas externalizados, problemas internalizados, depresión, problemas de conducta, somatización, actitud negativa hacia el colegio, actitud negativa hacia los padres, locus de control, estrés social, sentido de incapacidad). Se utilizó el autoinforme para niños o adolescentes. Los resultados normativos para cada escala presentan puntuaciones $\mathrm{T}$ estandarizadas con una media de 50 y una desviación estándar de 10.

Para valorar las estrategias de interacción, se utilizó la entrevista sobre el Conocimiento de Estrategias e Interacción con los Compañeros (CEIC) de DíazAguado (1995), que evalúa el conocimiento que el niño o la niña tiene de las estrategias para interactuar con sus iguales. La prueba consiste en cuatro historias que se presentan de manera oral y gráficamente, en torno a los siguientes contenidos: a) iniciar una relación; b) recuperar un objeto; c) convencer para un juego; y d) convencer para conseguir un objeto que es propiedad del otro niño. La evaluación de las estrategias se realiza teniendo en cuenta cuatro dimensiones del conocimiento social: elaboración, eficacia, consecuencias sociales y asertividad.

\section{Procedimiento}

Se pidió autorización al Instituto de Educación Básica del Estado de Morelos (IEBEM) para difundir la convocatoria dentro de los sectores de primaria. El sector autorizado para dicha convocatorio fue la Zona 5, donde realizó una presentación con directivos y encargados de de las Unidades de Servicio de Apoyo a la Escuela Regular (USAER), los cuales dieron la información de dicha invitación voluntaria a los padres de familia de niños y niñas detectados con altas capacidades.

Después, se reunió a los padres de familia de estos niños y niñas y se presentó el de manera general el objetivo y las temáticas del programa.

Aplicación de instrumentos de evaluación a los participantes. Previo a la evaluación, los padres de familia firmaron un consentimiento informado, en donde se le explica los fines de la investigación y se les pide su aval para llevarla a cabo con sus hijos.

Se dedicó una sesión de evaluación con los participantes, en dicha sesión se aplicaron los instrumentos que miden habilidades sociales $\mathrm{e}$ interacción de manera individual.

Además de presentar los datos de cada niño en cada una de las medidas, se procedió a examinar las relaciones entre ambas, mediante análisis de correlación por medido del coeficiente de correlación de Pearson. Los análisis estadísticos han sido realizados con el paquete estadístico informatizado SPSS v. 18.

\section{Resultados}

A continuación se muestran los resultados del BASC. En las escalas clínicas (desajuste clínico y desajuste social) a mayor puntuación se considera más grande la probabilidad de alarma (puntuaciones $\mathrm{T}$ entre 60 y 69 están en riesgo y de 70 o más, son clínicamente significativas). Es importante mencionar que dentro de la prueba existen unas escalas de control, las cuales hay que revisar para confirmar la validez de las respuestas. En ese sentido es importante mencionar que el participante número 1 no cumplió con los criterios de la escalas de control con respecto a la validez de las respuestas del BASC, por lo que no se analizarán sus respuestas en este instrumento.

Partiendo de lo anterior, dentro de las escalas clínicas las puntuaciones de los niños nos indican que se presentan algunos desajustes que hay que atender (ver Tablas 1 y 2).

Tabla 1.

Resultados de las escalas clínicas BASC (1 ${ }^{\circ}$ parte)

\begin{tabular}{|c|c|c|c|c|c|c|c|c|}
\hline \multirow{3}{*}{$\begin{array}{c}\text { Sujeto } \\
N=5\end{array}$} & \multicolumn{8}{|c|}{ Escalas clínicas } \\
\hline & \multicolumn{2}{|c|}{$\begin{array}{c}\text { Actitud } \\
\text { negativa } \\
\text { colegio }\end{array}$} & \multicolumn{2}{|c|}{$\begin{array}{c}\text { Actitud } \\
\text { negativa } \\
\text { profesores }\end{array}$} & \multicolumn{2}{|c|}{ Atipicidad } & \multicolumn{2}{|c|}{$\begin{array}{c}\text { Locus de } \\
\text { control }\end{array}$} \\
\hline & $\mathrm{T}$ & PC & $\mathrm{T}$ & PC & $\mathrm{T}$ & PC & $\mathrm{T}$ & PC \\
\hline 1 & - & - & - & - & - & - & - & - \\
\hline 2 & 53 & 76 & 61 & 87 & 51 & 64 & 59 & 83 \\
\hline 3 & 58 & 83 & 55 & 78 & 73 & 97 & 56 & 78 \\
\hline 4 & 48 & 61 & 55 & 78 & 77 & 98 & 50 & 57 \\
\hline 5 & 53 & 76 & 49 & 59 & 77 & 98 & 63 & 87 \\
\hline
\end{tabular}

Nota: Clasificación Típica $(\mathrm{T})$ de las escalas clínicas: Clínicamente significativo (70 o más), En riesgo (60-69), Medio (41-59), Bajo (31-40) y Muy bajo (30 o menos).

Tabla 2.

Resultados de las escalas clínicas BASC ( $2^{\circ}$ parte)

\begin{tabular}{|c|c|c|c|c|c|c|c|c|}
\hline \multirow{3}{*}{$\begin{array}{c}\text { Sujeto } \\
\mathrm{N}=5\end{array}$} & \multicolumn{8}{|c|}{ Escalas clínicas } \\
\hline & \multicolumn{2}{|c|}{$\begin{array}{l}\text { Estrés } \\
\text { social }\end{array}$} & \multicolumn{2}{|c|}{ Ansiedad } & \multicolumn{2}{|c|}{ Depresión } & \multicolumn{2}{|c|}{$\begin{array}{l}\text { Sentido de } \\
\text { incapacidad }\end{array}$} \\
\hline & $\mathrm{T}$ & $\mathrm{PC}$ & $\mathrm{T}$ & $\mathrm{PC}$ & $\mathrm{T}$ & $\mathrm{PC}$ & $\mathrm{T}$ & $\mathrm{PC}$ \\
\hline 1 & - & - & - & - & - & - & - & - \\
\hline 2 & 67 & 92 & 45 & 33 & 59 & 86 & 50 & 62 \\
\hline 3 & 62 & 88 & 53 & 62 & 55 & 82 & 50 & 62 \\
\hline 4 & 67 & 92 & 65 & 93 & 55 & 82 & 55 & 75 \\
\hline 5 & 53 & 70 & 55 & 70 & 67 & 92 & 60 & 83 \\
\hline
\end{tabular}

Nota: Clasificación Típica $(\mathrm{T})$ de las escalas clínicas: Clínicamente significativo (70 o más), En riesgo (60-69), Medio (41-59), Bajo (31-40) y Muy bajo (30 o menos).

Por un lado, las escalas que muestran a un número importante de participantes en riesgo o clínicamente significativo son estrés social y atipicidad. El estrés social se refiere a las sensaciones de tensión en sus interacciones con los demás. Estos niños pueden mostrar problemas relacionados con la ansiedad, quejas somáticas y pueden tener episodios de irritabilidad excesiva. En este caso, algunos de ellos presentan una puntuación $\mathrm{T}$ en el rango de riesgo (sujetos 2, 3, 4). Con respecto a la atipicidad, puede asociarse con algunas formas de conducta antisocial. Según el manual del BASC, estas conductas en niños pueden estar más asociadas con timidez y conducta introvertida. En este caso, tres sujetos $(3,4$ y 5 ) tienen una $\mathrm{T}$ superior a 70 lo cual puede indicar un trastorno emocional severo, como ansiedad e impulsividad.

Asimismo, otras escalas que requieren una atención 
particular por encontrarse en zona de riesgo son la de depresión (sujeto 5) y actitud negativa a los profesores (sujeto 2). Estas última escala forma parte importante en la vida de los niños. Nos puede mostrar su insatisfacción en relación con su experiencia escolar y la percepción general que los profesores como injustos o que no están motivados para ayudar a los estudiantes.

De manera general se podría decir que el niño 5 es el que presenta mayor desajuste escolar y clínico.

Por otro lado, en la Tabla 3 se observan los resultados de las escalas adaptativas (adaptación personal), las cuales hacen referencia a los aspectos socioafectivos y de relación con los otros. Una puntuación entre 31 y 40 nos indica que están en rango de riesgo. Los resultados muestran puntuaciones bastante heterogéneas entre los participantes. Se puede destacar que la mayoría de ellos está a más de dos desviaciones típicas de la puntuación media ( $\mathrm{T}$ 50) en la escala de relaciones interpersonales y los coloca en el rango de riesgo (sujeto 3) o clínicamente significativo (sujetos 2 y 5). Así mismo, en la escala de autoestima se destaca que algunos están en los rangos de riesgo (sujetos 2) o clínicamente significativo (sujeto 5). A su vez, la categoría de confianza en sí mismo ofrece un patrón similar en la mayoría de los niños, solo el 5 presenta una puntuación clínicamente significativa, con puntuaciones inferiores. Los niños con mejor ajuste personal son los sujetos 3 y 4; con peor ajuste, encontramos al sujeto 5 quien muestra todas las puntuaciones en el rango de clínicamente significativo.

Tabla 3.

Resultados de las escalas adaptativas BASC

\begin{tabular}{ccccccccc}
\hline $\begin{array}{c}\text { Sujeto } \\
\text { N=5 }\end{array}$ & $\begin{array}{c}\text { Relac. } \\
\text { Interpers. }\end{array}$ & \multicolumn{3}{c}{$\begin{array}{c}\text { Escalas adaptativas } \\
\text { Relaciones } \\
\text { con padres }\end{array}$} & Autoestima & \multicolumn{2}{c}{$\begin{array}{c}\text { Confianza } \\
\text { sí mismo }\end{array}$} \\
\hline & T & PC & T & PC & T & PC & T & PC \\
1 & - & - & - & - & - & - & - & - \\
2 & $\mathbf{6}$ & 1 & $\mathbf{1 6}$ & 2 & $\mathbf{3 9}$ & 13 & 49 & 38 \\
3 & $\mathbf{3 0}$ & 60 & 49 & 34 & 55 & 66 & 44 & 21 \\
4 & 43 & 13 & 41 & 14 & 55 & 66 & 44 & 21 \\
5 & $\mathbf{6}$ & 1 & $\mathbf{2 4}$ & 3 & $\mathbf{4 7}$ & 24 & $\mathbf{3 3}$ & 7 \\
\hline
\end{tabular}

Nota: Clasificación Típica (T) de las escalas adaptativas: Muy alto (70 o más), Alto (60-69), Medio (41-59), En riesgo (31-40) y Clínicamente significativo (30 o menos).

Por otro lado, con respecto a la entrevista sobre el conocimiento de estrategias de interacción (CEIC), se obtuvieron los siguientes resultados por niño (ver tabla 4).

Tabla 4.

Resultados del CEIC

\begin{tabular}{ccccccccc}
\hline $\begin{array}{c}\text { Sujeto } \\
\text { N=5 }\end{array}$ & Elaboración & Eficacia & \multicolumn{2}{c}{$\begin{array}{c}\text { Consec. } \\
\text { positiva } \\
\text { relación }\end{array}$} & Asertividad \\
\hline & PD & PC & PD & PC & PD & PC & PD & PC \\
\hline 1 & 19 & 71 & 24 & 94 & 25 & 96 & 18 & $\mathbf{3 0}$ \\
2 & 21 & 85 & 25 & 99 & 25 & 96 & 18 & $\mathbf{3 0}$ \\
3 & 21 & 85 & 24 & 94 & 22 & 77 & 18 & $\mathbf{3 0}$ \\
4 & $\mathbf{1 3}$ & $\mathbf{1 3}$ & $\mathbf{1 4}$ & $\mathbf{1 2}$ & $\mathbf{1 5}$ & $\mathbf{7}$ & $\mathbf{2 3}$ & 68 \\
5 & $\mathbf{9}$ & $\mathbf{0}$ & $\mathbf{1 1}$ & $\mathbf{1}$ & $\mathbf{1 1}$ & $\mathbf{0}$ & $\mathbf{7}$ & $\mathbf{0}$ \\
\hline
\end{tabular}

Como se puede observar, la mayoría de los participantes conocen estrategias para establecer una adecuada relación con el otro y la satisfacción de necesidades personales. Sin embargo, en la estrategia de asertividad las puntuaciones son inferiores en la mayoría de los participantes; lo cual nos indica que a pesar de que sus estrategias de interacción son adecuadas y eficaces, la falta de claridad en los ideales o metas, podría generar pérdida de autoestima y confianza en sí mismos.

A su vez, podemos ver que dos de los cinco participantes (4y 5) obtienen puntuaciones inferiores en la mayoría de las estrategias. Uno de ellos (4) llega a ser asertivo, lo cual nos indica que obtiene los resultados esperados, pero sus estrategias de interacción no son adecuadas, ya que consigue sus objetivos a partir de golpes, berrinches o peleas. A su vez, el otro participante (5), muestra puntuaciones muy inferiores a lo esperado en todas las dimensiones.

Con estos datos podemos concluir que los participantes 1, 4 y 5 requieren de una atención inmediata dado que presentan desajustes personales importantes que pudieran afectar su tránsito por la escuela y en su vida personal. En este caso, el participante 1 muestra un desajuste en la adaptación en el ámbito escolar, lo cual se pone de manifiesto por una actitud negativa hacia el colegio y hacia los profesores; además muestra un bajo nivel en sus relaciones interpersonales y alto estrés social.

Por otro lado, para analizar si ambas medidas (habilidades socioafectivas e interacción) tenían alguna relación, realizamos un análisis de correlación a través del coeficiente de correlación de Pearson, encontrando lo siguiente. Correlaciones entre algunas dimensiones del CEIC: Consecuencias positivas para la relación con dos dimensiones del mismo instrumento elaboración ( $r=$ $.954, p<.05)$ y eficacia $(r=.983, p<.01)$, ambas con valencia positiva. Así mismo, se encuentra una correlación alta positiva entre las dimensiones elaboración y eficacia $(r=.985, p<.01)$. Por otro lado, se observa una correlación moderada entre las siguientes escalas del BASC: estrés social y confianza en sí mismo $(r=.924, p<.05)$, depresión y autoestima $(r=-0.954$, $p<.05$ ), esta última con valencia negativa, es decir, a mayor depresión, menor autoestima. A su vez, se encuentran correlaciones moderadas entre algunas dimensiones del CEIC y escalas del BASC, por ejemplo: asertividad y confianza en sí mismo ( $r=.907, p<.05)$, Asertividad y estrés social $(r=.929, p<.05)$ y elaboración y sentido de incapacidad ( $r=-.913, p<.05$ ), esta última con valencia negativa, es decir, a menor elaboración mayor sentido de incapacidad.

\section{Discusión}

En este estudio realizamos un análisis de las características socioafectivas de los niños con altas capacidades intelectuales al inicio de su participación en un programa extracurricular. Los participantes muestran diferentes niveles de adaptación social y afectiva, mostrando algunos rasgos de desajuste personal o social en la mayoría de ellos; particularmente tres participantes presentan características que los sitúan en riesgo. Pobres relaciones interpersonales, baja autoestima, estrés social, aunado a un deficiente conocimiento de estrategias 
adecuadas de interacción son solo algunas dimensiones sobre las que hay que trabajar seriamente, ya que se ha comprobado que las relaciones positivas de los niños con sus iguales dependen en gran medida de su capacidad para conocer y desplegar una variedad de características sociocognitivas tales como adoptar distintas perspectivas sociales, el concepto de amistad, el conocimiento interpersonal y los objetivos sociales, entre otros (DíazAguado, 1995). También hay que tener en cuenta las limitaciones del estudio. Con estos resultados no se puede generalizar que los alumnos con altas capacidades son inadaptados $\mathrm{y}$ presentan baja autoestima $\mathrm{y}$ dificultades para relacionarse en general, dado que la muestra tenía el sesgo de la forma en que fueron invitados a participar. Pero sí sería oportuno señalar que para que no se produzcan estas dificultades hay que trabajar de forma conjunta con todos los agentes educativos, para proponer acciones que hagan florecer los talentos de los niños y niñas, y no los disminuyan. Programas como el PIPAC, en el que se trabajan los ejes de habilidades intrapersonales, interpersonales y de trabajo colaborativo parecen ser una oportunidad para dar una respuesta educativa adecuada.

\section{Referencias}

Belda, V. (2012). Alumnado con altas capacidades intelectuales: conceptualización, identificación e intervención desde el marco escolar y familiar. Tesis de maestría, Universidad Internacional de la Rioja, España.

http://reunir.unir.net/bitstream/handle/123456789/170/ TFG\%20Belda\%20Ribera.pdf?sequence $=1$

Benito, Y. (2000). Intervención e investigación psicoeducativas en alumnos superdotados. Salamanca: Amarú.

Borges, A., Hernández-Jorge, C. \& Rodríguez-Naveiras, E. (2011). Evidencias contra el mito de la inadaptación de las personas con altas capacidades intelectuales. Psicothema, 23(3), 362-367.

Castro, M. (2008). Niños con altas capacidades intelectuales ¿Niños en riesgo social? Educación y Futuro, 18, 163-176. Recuperado de: http://dialnet.unirioja.es/servlet/articulo?codigo=2572 085

Clemente, R., Regal, R., Górriz, A. \& Villanueva, L. (2003). Interacción social entre iguales: comprensión de normas socioconvencionales y morales. Actas de las Jornadas de Fomento a la Investigación: Universidad Jaume I. (pp. 2-11). Recuperado de: http://sic.uji.es/bin/publ/edicions/jfi8/psi/19.pdf

Comes, G., Díaz, E., Luque, A. \& Moliner, O. (2008). La evaluación psicopedagógica del alumnado con altas capacidades intelectuales. Revista de Educación Inclusiva, 1, 103-117. Recuperado de: http://www.ujaen.es/revista/rei/linked/documentos/do cumentos/8.pdf

Díaz-Aguado, M. J. (1986). El papel de la interacción entre iguales en la adaptación escolar y el desarrollo social. Madrid: Centro de Investigación Educativa.
Díaz-Aguado, M. J. (1995). Niños con dificultades socioemocionales. Instrumentos de evaluación. Madrid: Ministerios de Asuntos Sociales.

Ferrándiz, C., Prieto, Ma., Fernández, Ma., Soto, G., Ferrando, M. \& Badía, Ma. (2010). Modelo de identificación de alumnos con altas habilidades de Educación Secundaria. Revista Electrónica Interuniversitaria de Formación del Profesorado, 13(1), 63-74. Recuperado de: http://www.aufop.com/aufop/uploaded_files/articulos/ 1268616568.pdf

Flanagan, A. \& Arancibia, V. (2005). Talento académico: un análisis de la identificación de alumnos talentosos efectuada por profesores. Psykhe, 14(1), 121-135. Recuperado de: http://redalyc.uaemex.mx/pdf/967/96714110.pdf

Flores, N. \& Santoyo, C. (2009). Estabilidad de cambio de las relaciones sociales entre niños. Análisis de mecanismos funcionales. Revista Mexicana de Análisis de la Conducta, 35 (1), 59-74. Recuperado de: www.revistas.unam.mx/index.php/rmac/article/downl oad/398/15483

García, J. (1993). Interacción entre iguales en entorno de integración escolar. Tesis doctoral, Departamento de Didáctica y Organización Escolar, Universidad Complutense de Madrid, Madrid, España. http://biblioteca.ucm.es/tesis/19911996/S/5/S5004601. pdf

Goldrine, T. \& Rojas, S. (2007). Descripción de la práctica docente a través de la interactividad profesoralumnos. Estudios Pedagógicos, 33(2), 177- 197. Recuperado http://www.scielo.cl/pdf/estped/v33n2/art10.pdf

Gómez, M. \& Valadez, M. (2010). Relaciones de la familia y del hijo con superdotación intelectual. Faísca, 15(17), 67-85.

Hernández, C. \& Borges, A. (2010). Entorno del alumnado de altas capacidades intelectuales frente a sus compañeros de distinto nivel de inteligencia Faísca, 15 (17), 36-49.

López, M. (2008). Estudio, mito y realidad del niño sobredotado. México: Trillas.

Lovecky, D. (1995). Highly gifted children and peer relationships. Counseling and Guidance Newsletter National Association for Gifted Children (NAGC), 5(3), 2-7. Recuperado de: http://www.davidsongifted.org/db/Articles_id_10129. aspx

Narea, M., Lissi, M. \& Arancibia, V. (2006). Impacto en la sala de clases de un programa extracurricular para alumnos con talentos académicos. Psykhe, 15(2), 8192. Recuperado de: http://www.scielo.cl/scielo.php?pid=S071822282006000200008\&script=sci_arttext

Reynold, C. \& Kamphaus, R. (2004). Sistema de evaluación de la conducta de niños y adolescentes (BASC). Madrid: TEA.

Roedell, W. (2011). Vulnerabilities of highly gifted children. Roeper Review, 6(1), 127-130. 
Vialle, W., Heaven, P. \& Ciarrochi, J. (2007). On Being Gifted, but Sad and Misunderstood: Social, emotional, and academic outcomes of gifted students in the Wollongong Youth Study. Educational Research and Evaluation, 13(6), 569-586. doi: 10.1080/13803610701786046

\section{Agradecimientos}

Este trabajo fue realizado gracias a los fondos de la convocatoria UAEM/PIDE 2013 